\title{
Management of culture positive urinary tract infections in children aged 0-16 years: An audit conducted in a District General Hospital in the United Kingdom
}

\author{
*S Jeyabalan ${ }^{1}$, E. Ikpakwu², G Liyanage ${ }^{3}$ \\ Sri Lanka Journal of Child Health, 2016; 45(3): 172-176
}

\begin{abstract}
Background: Urinary tract infection (UTI) is common among children. Management of UTI requires prompt diagnosis, treatment and most often, a regular follow up.

Objectives: To evaluate compliance with the NICE guidelines 2007 when managing children aged 0 to16 years with bacteriologically proven UTI in a District General Hospital.
\end{abstract}

Method: A descriptive retrospective study was carried out at Bronglais General Hospital, Aberystwyth, United Kingdom (UK) on children aged 0 to 16 years with culture positive UTI from $1^{\text {st }}$ January 2012 to $31^{\text {st }}$ December 2014. Infants and children with urinary catheters in situ, pre-existing renal disorders and immune-suppression were excluded from the study. Patients were grouped into age categories to facilitate comparison of treatment and radiological investigations. Further categorisation was done according to NICE guidelines for UTI in children. Approval for the conduct of the audit was given by the Clinical Audit Department, Bronglais Hospital, UK. Ethical clearance for the study was obtained from the Ethical Review Committee of the Sri Lanka College of Paediatricians.

${ }_{1}^{1}$ Acting Consultant Paediatrician, Teaching Hospital Jaffna, Sri Lanka, ${ }^{2}$ Consultant Paediatrician Bronglais General Hospital, Aberystwyth, UK, ${ }^{3}$ Senior Lecturer, Dept. of Paediatrics, Faculty of Medical Sciences, University of Sri Jayewardenepura and Honorary Consultant Paediatrician, Colombo South Teaching Hospital, Sri Lanka

*Correspondence: dr_sbalan@yahoo.com

(Received on 01 September 2015: Accepted after revision on 23 October 2015)

The authors declare that there are no conflicts of interest

Personal funding was used for this project.

Open Access Article published under the Creative

Commons Attribution CC-BY (CC) (i)

Results: There were 104 children aged 0 to 16 years with culture proven UTI during the study period of whom 8 were excluded. Only $25 \%$ of infants 3 months old or less received intravenous (IV) antibiotics in compliance with the guidelines; $25 \%$ had only oral antibiotics and $50 \%$ had IV followed by oral antibiotics. For treatment of lower tract infection, duration of antibiotics prescription was longer than recommended. Overall adherence to investigations such as ultrasonography, dimercaptosuccinic acid scan and micturating cystourethrogram was $81 \%$, $91 \%$ and $93 \%$ respectively. All children with recurrent UTIs were prescribed antibiotic prophylaxis adhering to NICE guidelines.

Conclusions: Duration of antibiotic therapy in UTI was in compliance with NICE guidelines in 16-86\% children. Radiological investigations were requested in UTI in compliance with NICE guidelines in 7197\% children. Prophylactic antibiotics were prescribed in UTI in compliance with NICE guidelines in $34-100 \%$ children.

DOI: http://dx.doi.org/10.4038/sljch.v45i3.8142

(Key words: Urinary tract infection, children, antibiotics)

\section{Introduction}

Urinary tract infections (UTIs) occur throughout childhood, with the highest frequency in infancy ${ }^{1,2}$. The male to female ratio is 1.85: 1 in infants ${ }^{3}$. However, in preschool and school-aged children, the prevalence of UTI is about $1-5 \%$ for females and UTI is rare in males. Following puberty its incidence increases in females but in males UTI remains uncommon $^{2}$. Infections may involve both lower and upper urinary tracts. Organisms causing UTI include Escherichia coli, Klebsiella, Enterobacter, Proteus, Pseudomonas and Enterococci. Escherichia coli accounts for $85-90 \%$ of all infections in this age group $^{2}$. Diagnosis of UTI depends on analysis of an uncontaminated specimen. Urine dipstick/urine full report is useful for screening as well as being a diagnostic tool, whereas urine culture is considered the "gold standard" for diagnosis ${ }^{1,4}$. Management of 
UTI in infants and children requires follow up with appropriate radiological investigations ${ }^{4,5}$. The ultimate goal is the preservation of kidney function. Guidelines for management have been revised several times over the past decade. Threshold for radiological imaging such as micturating cystourethrogram (MCUG) and dimercaptosuccinic acid (DMSA) scan is high in current practice. The role of antibiotic prophylaxis has been questioned by a number of meta-analyses ${ }^{6}$. According to Nice Guidelines 2007, antibiotic prophylaxis should not be routinely recommended in infants and children following a first-time UTI. It may be considered in infants and children with recurrent infections ${ }^{5}$.

\section{Objective}

To evaluate compliance with the NICE guidelines 2007 when managing children aged 0 to 16 years with bacteriologically proven UTI in a District General Hospital.

\section{Method}

A descriptive retrospective study was carried out at Bronglais General Hospital, Aberystwyth, United Kingdom on children aged 0 to 16 years with culture proven UTI. Data were obtained from electronic records of children between 0 to 16 years with a diagnosis of a UTI from $1^{\text {st }}$ January 2012 to $31^{\text {st }}$ December 2014, a period of 3 years. Infants and children with urinary catheters in situ, pre-existing renal disorders and immune-suppression were excluded from the study. "Culture proven UTI" was defined as at least one culture of urine of a known urinary pathogen along with symptoms of UTI such as fever, haematuria, loin pain, dysuria, frequency or incontinence. Positive urine culture was defined as a growth of single bacterial organism at $\geq 10^{8}$ colony forming units $(\mathrm{cfu}) / 1$ in a clean catch, midstream urine sample or growth of single bacteria at $\geq 10^{7} \mathrm{cfu} / 1$ from a catheter specimen from a child with fever or any growth from a supra-pubic specimen. Patients were grouped into age categories to facilitate comparison of treatment and radiological investigations. Further categorisation was done according to NICE guidelines for UTI in children ${ }^{5}$. The relevant portions of the NICE guidelines applicable to the current audit are given below:

\section{Antibiotic route and duration for acute infection}

1. Infants younger than 3 months with a possible UTI should be referred immediately to the care of a paediatric specialist. Treatment should be with parenteral antibiotics.
2. For infants and children 3 months or older with acute pyelonephritis/upper UTI:

- Consider referral to a paediatric specialist

- Treat with oral antibiotics for 7-10 days. Use of an oral antibiotic with low resistance patterns is recommended, for example cephalosporin or co-amoxiclav

- If oral antibiotics cannot be used, treat with an intravenous (IV) antibiotic such as cefotaxime or ceftriaxone for $2-4$ days followed by oral antibiotics for a total duration of 10 days.

3. For infants and children 3 months or older with cystitis/lower UTI:

- Treat with oral antibiotics for 3 days. Choice of antibiotics should be directed by locally developed multidisciplinary guidance. Trimethoprim, nitrofurantoin, cephalosporin or amoxicillin may be suitable.

- The parents or carers should be advised to bring the infant or child for reassessment if the infant or child is still unwell after 24-48 hours. If an alternative diagnosis is not made, a urine sample should be sent for culture to identify the presence of bacteria and determine antibiotic sensitivity if urine culture has not already been carried out.

4. For infants and children who receive aminoglycosides (gentamicin or amikacin), once daily dosing is recommended.

5. If parenteral treatment is required and IV treatment is not possible, intramuscular treatment should be considered.

6. If an infant or child is receiving prophylactic medication and develops an infection, treatment should be with a different antibiotic, not a higher dose of the same antibiotic.

7. Asymptomatic bacteriuria in infants and children should not be treated with antibiotics.

8. Laboratories should monitor resistance patterns of urinary pathogens and make this information routinely available to prescribers.

\section{Antibiotic prophylaxis}

1. Antibiotic prophylaxis should not be routinely recommended in infants and children following first-time UTI. 
2. Antibiotic prophylaxis may be considered in infants and children with recurrent UTI.

3. Asymptomatic bacteriuria in infants and children should not be treated with prophylactic antibiotics.

\section{Radiological investigations}

1. Infants and children with atypical UTI should have ultrasound of the urinary tract during the acute infection to identify structural abnormalities of the urinary tract such as obstruction. This is to ensure prompt management. Atypical UTI includes seriously ill children, poor urine flow, abdominal or bladder mass, raised creatinine, septicaemia, failure to respond to treatment with suitable antibiotics within 48 hours and infection with non-Escherichia coli organisms.

2. For infants younger than 6 months with first-time UTI that responds to treatment, ultrasound should be carried out within 6 weeks of the UTI.

3. For infants and children aged 6 months and older with first-time UTI that responds to treatment, routine ultrasound is not recommended unless the infant or child has atypical UTI.

4. Infants and children who have had a lower UTI should undergo ultrasound (within 6 weeks) only if they are younger than 6 months or have had recurrent infections. Recurrent UTI is defined as two or more episodes of UTI with acute pyelonephritis/ upper UTI or one episode of UTI with acute pyelonephritis/upper UTI plus one or more episode of UTI with cystitis/lower UTI or three or more episodes of UTI with cystitis/lower UTI.

5. A DMSA scan 4-6 months following the acute infection should be used to detect renal parenchymal defects.

6. If the infant or child has a subsequent UTI while awaiting DMSA, the timing of the
DMSA should be reviewed and consideration given to doing it sooner.

7. Routine imaging to identify VUR is not recommended for infants and children who have had a UTI.

8. When a micturating cystourethrogram (MCUG) is performed, prophylactic antibiotics should be given orally for 3 days with MCUG taking place on the second day.

Approval for the conduct of the audit was given by the Clinical Audit Department, Bronglais Hospital, UK on $18^{\text {th }}$ December 2014. Ethical clearance for the study was obtained from the Ethical Review Committee of the Sri Lanka College of Paediatricians on $20^{\text {th }}$ March 2015.

\section{Results}

There were 104 children aged 0 to 16 years with culture proven UTI during the study period of whom 8 were excluded due to exclusion criteria. Records of 96 children between 0 to 16 years of age included in the study were analysed. In the 8 children aged 3 months or less, 6 were male giving a male: female ratio of $3: 1$. In the 88 children aged more than 3 months, 68 were female giving a male: female ratio of 1:3.4. In children more than 3 years of age, 58 had lower UTI, 30 had upper UTI and 14 had recurrent UTI. The commonest causative pathogen was Escherichia coli (84\%). Klebsiella, Proteus and Pseudomonas were the other organisms isolated. Fifty percent of the infants aged 3 months or less received IV antibiotics initially followed by oral antibiotics, $25 \%$ were treated with IV antibiotics alone and $25 \%$ had oral antibiotics alone. Majority $(80 \%)$ of children more than 3 months received oral antibiotics.

Table 1 shows the duration of antibiotic therapy for different age groups. There was a considerable variation in the duration of antibiotic therapy. Table 2 shows the radiological investigations requested in UTI (inclusive of radiological investigations performed during or after previous episodes)

Table 1: Duration of antibiotic therapy

\begin{tabular}{|l|c|c|c|c|}
\hline \multicolumn{1}{|c|}{ Age } & $\begin{array}{c}\text { 3 days } \\
\text { No. (\%) }\end{array}$ & $\begin{array}{c}\mathbf{5} \text { days } \\
\text { No. (\%) }\end{array}$ & $\begin{array}{c}\mathbf{7 - 1 0} \text { days } \\
\text { No. (\%) }\end{array}$ & $\begin{array}{c}\text { Compliance with } \\
\text { guidelines No. (\%) }\end{array}$ \\
\hline 3 months or less & $01(12.5)$ & $02(25.0)$ & $05(62.5)$ & $05(63.0)$ \\
\hline More than 3 months & & & & \\
Lower urinary tract infection & $09(16.0)$ & $26(45.0)$ & $23(39.0)$ & $09(16.0)$ \\
Upper urinary tract infection & - & $04(14.0)$ & $26(86.0)$ & $26(86.0)$ \\
\hline
\end{tabular}


Table 2: Radiological investigations requested in urinary tract infection

\begin{tabular}{|l|c|c|c|c|}
\hline & $\begin{array}{c}\text { Responded well } \\
\text { to treatment }\end{array}$ & Atypical & Recurrent & $\begin{array}{c}\text { Compliance with } \\
\text { guidelines No. (\%) }\end{array}$ \\
\hline Ultrasound scan during acute infection & 12 & 12 & 07 & $79(82)$ \\
\hline Ultrasound scan within 6 weeks & 24 & - & 07 & $68(71)$ \\
\hline DMSA scan after 4-6 months & 02 & 08 & 11 & $91(95)$ \\
\hline Micturating cystourethrogram & - & 02 & 03 & $93(97)$ \\
\hline
\end{tabular}

DMSA: Dimercaptosuccinic acid

All patients aged 3 months or less with atypical UTI have undergone USS during the acute stage and DMSA scan 4-6 months later. Thirty three percent of children between 3 months to 16 years with uncomplicated UTI have had an USS as a routine investigation that is not recommended in the guidelines. Prescription of prophylaxis is summarized in table 3. All patients with recurrent UTI were given prophylaxis.

Table 3: Prophylactic antibiotics prescribed

\begin{tabular}{|l|c|c|c|}
\hline & $\begin{array}{c}\text { Prophylaxis } \\
\text { given }\end{array}$ & $\begin{array}{c}\text { Prophylaxis not } \\
\text { given }\end{array}$ & $\begin{array}{c}\text { Compliance with guidelines } \\
\text { No. (\%) }\end{array}$ \\
\hline Upper tract infection & 04 & 26 & $26(87)$ \\
\hline Lower tract infection & - & 58 & $58(100)$ \\
\hline UTI that responded well to treatment & 02 & 10 & $10(84)$ \\
\hline Atypical UTI & 08 & 04 & $04(34)$ \\
\hline Recurrent UTI & 14 & - & $14(100)$ \\
\hline Infants $\leq 3$ months & 02 & 06 & $06(75)$ \\
\hline
\end{tabular}

\section{UTI: urinary tract infection}

\section{Discussion}

Local as well as international guidelines for the management of UTIs exist. However, these guidelines for UTI are frequently changing with new evidence. NICE is an organisation which carries out assessments of the most appropriate treatment regimens for different diseases taking desired medical outcomes and economic aspects into consideration. Many parts of the world refer to NICE guidelines for uniformity in the management based on best evidence.

Our findings show that Bronglais General Hospital, Aberystwyth, United Kingdom, there are deviations from guidelines. According to the guidelines, infants of 3 months or less should be treated with parenteral antibiotics. However, in our study, the route of administration of antibiotics did not have a uniform pattern. Several factors such as how unwell the child was during the infection, socioeconomic circumstances and willingness for long hospital stay could have been responsible for this. However, in our study, we have not assessed the impact of the above factors on reluctance. There was a significant digression from guidelines for duration of antibiotics. Time taken for the initial response to antibiotics could have prompted the clinician to extend the duration of antibiotics more than the specified period in the guidelines. Considering radiological investigations, we noted that the adherence to guidelines was substantial. This shows the awareness of the clinician on the latest guidelines. Antibiotic prophylaxis to prevent repeated UTI may be considered in infants and children with recurrent UTI. The potential benefit of preventing recurrent UTI with prophylaxis should be weighed against the risks of antimicrobial resistance. In our study, we have not evaluated the outcome of these patients. Assessment of outcome in a longitudinal study would be useful to confirm the efficacy and our dependability on these guidelines.

\section{Conclusions}

- Duration of antibiotic therapy in UTI was in compliance with NICE guidelines in 16$86 \%$ children.

- Radiological investigations were requested in UTI in compliance with NICE guidelines in $71-97 \%$ children. 
- Prophylactic antibiotics were prescribed in UTI in compliance with NICE guidelines in $34-100 \%$ children.

\section{References}

1. Zorc JJ, Kiddoo DA, Shaw KN. Diagnosis and management of paediatric urinary tract infections. Clinical Microbiology Reviews 2005; 18(2): 417-22.

http://dx.doi.org/10.1128/CMR.18.2.417422

.2005

PMid: 15831830 PMCid: PMC1082801

2. Dulczak S, Kirk J. Overview of the evaluation, diagnosis and management of urinary tract infections in infants and children. Urologic Nursing 2005; 25(3):18592.

PMid: 16050349

3. Urinary tract infections in children: EAU/ESPU Guidelines. 2015. Available from:

http://eu-acme.org/europeanurology
4. Bitsori M, Galanakis E. Paediatric urinary tract infections: diagnosis and treatment. Expert Review of Anti-Infective Therapy 2012; 10 (10):1153-64.

http://dx.doi.org/10.1586/eri.12.99

PMid: 23199401

5. National Institute for Health and Clinical Excellence, Urinary tract infection in children: diagnosis, treatment and long-term management. 2007. Available from: http://www.nice.org.uk/guidance/cg54

6. Jadresic L. Urinary tract infection in children. Paediatrics and Child Health 2014; 24(7): 289-92.

http://dx.doi.org/10.1016/j.paed.2013.11.002 